\title{
Sideslip of the Medial Rectus Muscle during Vertical Eye Rotation
}

\author{
Kyoung-Min Lee, ${ }^{1,2}$ Annie P. Lai, ${ }^{3}$ James Brodale, ${ }^{1}$ and Arthur Jampolsky ${ }^{1}$
}

Purpose. The kinematics of eye rotation is not entirely elucidated despite two centuries of fascination with the deceptively simple yet geometrically complex nature of the movement. Recently, the traditional view that oculorotatory muscles except the superior oblique muscle exert straight pull on the globe has been challenged by the claim that the muscles also go through a connective tissue pulley-like structure that holds them steady during eye rotation. Although earlier studies failed to observe sideslippage at the posterior part of muscles, a finding supportive of the pulley hypothesis, the conclusions should not be taken as conclusive given short-comings in the techniques used in the studies.

Methods. The authors developed a novel method of image analysis to improve spatial resolution and applied the method for investigating the medial rectus muscle, the entire length of which can easily be identified in magnetic resonance images.

RESULTS. Contrary to previous reports, vertical sideslippage was observed at the posterior part of the muscle during vertical eye rotation between two tertiary eye positions. Furthermore, the sideslip varied as a function of horizontal eye position, in accordance with the half-angle rule of Listing's law.

Conclusions. These findings are more consistent with the traditional view of the restrained shortest-path model than with the pulley model and have further implications for basic and clinical understanding of ocular kinematics. (Invest Ophthalmol Vis Sci. 2007;48:4527-4533) DOI:10.1167/iovs.07-0496

$\mathrm{D}$ emer and colleagues ${ }^{1}$ put forth the proposal that all oculorotatory muscles, other than the superior oblique, go through connective tissue pulleys. The idea sprang from observations that the posterior part of muscles did not shift whereas the anterior part moved along with the globe. ${ }^{2,3}$ However, methodological limits in the latter studies require caution in accepting them as conclusive. Given that expected sideslips were small-no larger than a few millimeters ${ }^{3}$ - they might have been obscured by the techniques used for image analysis, which involved free-hand drawings on computed tomography/magnetic resonance imaging (MRI). ${ }^{2,3}$ Moreover, tertiary eye positions that would have maximized the sideslip

From the ${ }^{1}$ The Smith-Kettlewell Eye Research Institute, San Francisco, California; the ${ }^{2}$ Department of Neurology, Seoul National University, Seoul, South Korea; and the ${ }^{3}$ Bay Imaging Consultants, Berkeley, California

Supported by the Smith-Kettlewell Eye Research Institute and by the Brain Research Center of The 21st Century Frontier Research Program funded by the Ministry of Science and Technology of Republic of Korea (KML).

Submitted for publication April 24, 2007; revised June 1, 2007; accepted July 16, 2007.

Disclosure: K.-M. Lee, None; A.P. Lai, None; J. Brodale, None; A. Jampolsky, None

The publication costs of this article were defrayed in part by page charge payment. This article must therefore be marked "advertisement" in accordance with 18 U.S.C. $\$ 1734$ solely to indicate this fact

Corresponding author: Arthur Jampolsky, The Smith-Kettlewell Eye Research Institute, 2318 Fillmore Street, San Francisco, CA 94115; aj@ski.org. were not tested in one of the studies, and the other did not report on comparisons for individual subjects.

The pulley hypothesis ${ }^{1}$ and traditional views, such as the (restrained) shortest-path model, ${ }^{4-6}$ differ in their predictions about how the medial rectus (MR) muscle would change its position during vertical ocular rotations. The difference is schematically depicted in Figure 1. For vertical rotation of the eye, the axis of rotation lies in a horizontal plane, and the MR insertion draws an arc about the axis. The pulley hypothesis predicts that the muscle behind the pulley would not move vertically and would be held by the pulley, whereas the shortest-path model predicts vertical displacement at all parts of the muscle, with the amount proportional to proximity to the insertion.

The two models also differ regarding whether vertical sideslip would depend on horizontal eye position: The shortestpath model predicts such a dependency, but the pulley hypothesis does not. This difference is illustrated by the rows of panels in Figure 1. When vertical eye movements take place at different horizontal eye positions, for example, in abduction versus adduction, the rotational axis for the vertical rotations lies within the horizontal plane and tilts to the side by half the amount of the horizontal deviation of the eye from the primary position (the half-angle rule, a corollary of Listing's law). ${ }^{7}$ Now, because of this rule, the relative position of the MR insertion and the axis for vertical rotation changes as a function of horizontal eye position. The insertion, because it is attached to the globe, rotates fully with it, whereas the rotation axis rotates only half as much; the separation between the two, therefore, becomes greater as the eye assumes a more abducted position. Because the separation equals the radius of the arc the insertion draws during a vertical rotation, the size of the arc and the vertical shift of the MR will also be greater when the eye is more abducted. Dictated by these geometric considerations, the shortest-path model predicts that vertical sideslip is dependent on horizontal eye position (Fig. 1, left panels). In contrast, the pulley model would not predict such dependence on horizontal eye position because the pulleys are supposed to assume a constant vertical position during ocular rotation (Fig. 1, right panels). (More recent versions of the hypothesis, namely, the active pulley hypotheses, suggest a coordinated anterior-posterior movement of the pulley with the muscle contraction, but they still suppose the pulley to be fixed in the perpendicular direction, i.e., the vertical direction in the MR.)

In this study, we tested the two models by imaging orbital contents with high-resolution MRI while subjects maintained tertiary eye positions. Specifically, we asked whether the MR muscle shifted perpendicularly between tertiary eye positions that only differ vertically and whether the shifts varied with the horizontal component of the eye positions.

\section{Materials AND Methods}

$\mathrm{T}_{2}$-weighted images by 3D RARE (Turbo-SE) sequences were acquired with a 1.5-T scanner (Siemens, Munich, Germany) and a four-phase array surface coil while five healthy volunteers fixated a target at tertiary positions $\left(30^{\circ}\right.$ in horizontal and vertical deviations from the center, at a distance of approximately $30 \mathrm{~cm}$ ). All procedures were 
(restrained) shortest-path model

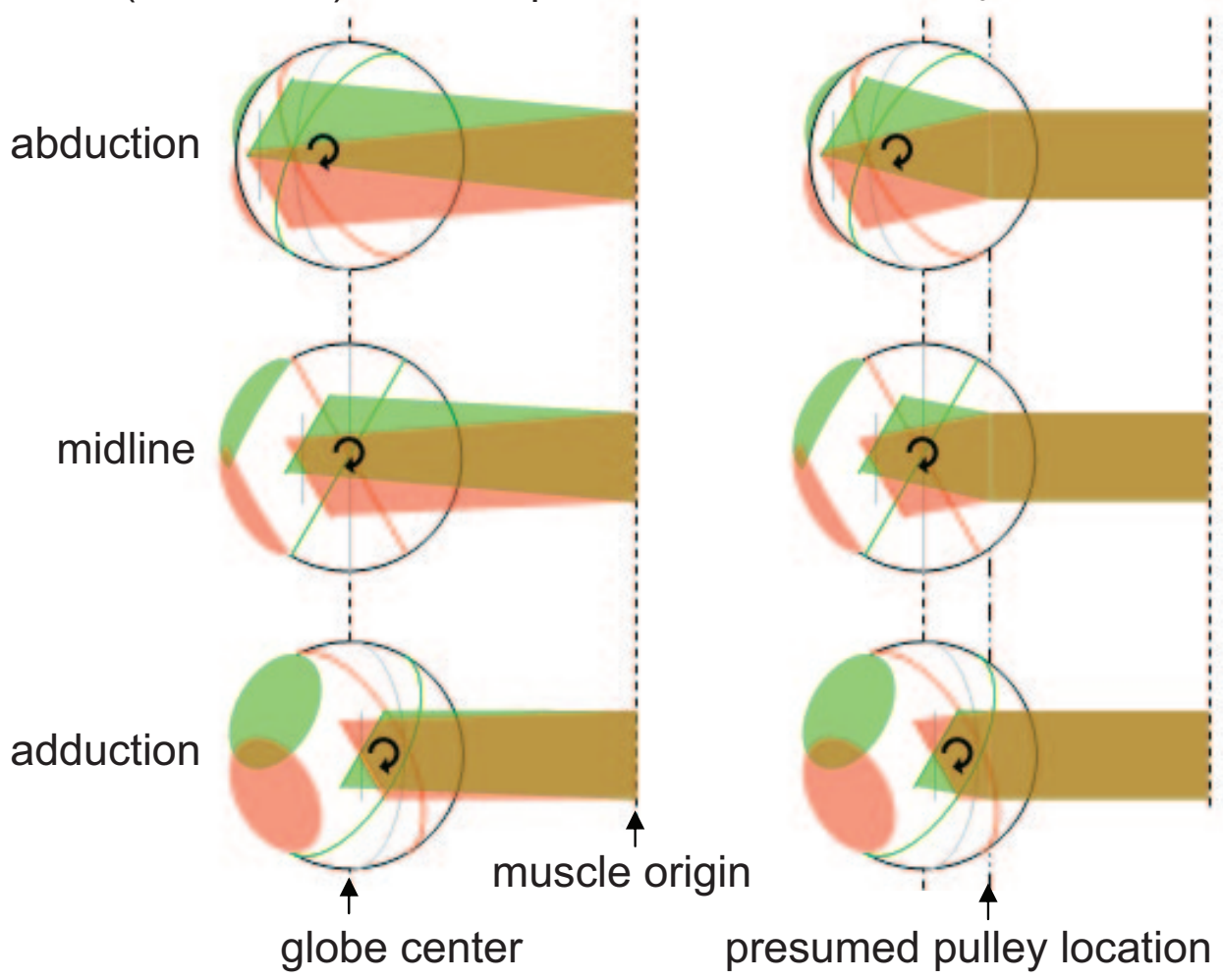

Figure 1. Schematic depictions of the globe and the MR muscle in two vertical eye positions. Drawings in the two positions are superimposed with the cornea; the globe equator and the muscle colored green for a supraduction position and red for infraduction. Portions of these structures that overlap in the two positions are shown in yellow. Configurations are separately shown for the eye in abduction (upper row), at midline (middle row), and in adduction (bottom row). Left: as predicted by the (restrained) shortest-path model, the MR muscle exerts a straight pull on the eyeball. Right: predictions by the pulley hypothesis. Details such as bending and wrapping around the globe of the insertion are omitted. The circular arrow in each panel denotes the location of rotational axis between the two eye positions. Note that the distance between the axis and the muscle insertion changes as a function of horizontal deviation because of the halfangle rule. carried out in strict adherence to the Declaration of Helsinki with informed consent obtained from each subject. Image acquisition was set in axial planes, and the field of view $(108 \mathrm{~mm} \times 108 \mathrm{~mm})$ included the orbit on the right side, the nasal cavity, and the retro-orbital intracranial structures, including parts of the temporal cortex. In-plane resolution of the images was $0.42 \mathrm{~mm} \times 0.42 \mathrm{~mm}$ (matrix size of $256 \times 265$ ), with slices separated by $0.375 \mathrm{~mm}$.

Image volumes were registered to correct against head motion using an algorithm ${ }^{8}$ as implemented in SPM2 (The Wellcome Department of Imaging Neuroscience, UCL, London, UK), a software package widely used for functional MRI data processing. The registration was performed after the intra-orbital portion in the volumes was masked out. Masking was performed because only head motion was to be corrected, though orbital contents had obviously moved between scans because of eye movements as well. The resultant transformation matrices correcting against head motion were then applied to the whole volumes for further analysis of changes caused by eye movements only.

To compute the amount of expected sideslip predicted by the shortest-path model, ${ }^{4,5}$ the following measurements were made using individual MRI data: horizontal and vertical eye positions at each MRI volume, axis for vertical rotation, location of MR insertion, and length of MR muscle (in front and back of the point of tangency). First, eye positions were deduced from the location of the lens with respect to the globe center, after the globe and lens were segmented out by taking advantage of the high contrast between the sclera and lens and the vitreous in the images. Second, because the torsion of the globe could not be measured from the MRI data, we made the assumption that the rotation axis was perpendicular to the midline for vertical rotations from the primary position. For rotations between two tertiary positions on the same side, the axis was assumed to tilt to the side by the half amount of horizontal components of the tertiary positions. Third, we presumed that the MR insertion laid $34^{\circ}$ anterior to the globe equator. ${ }^{5}$ (The equator was identified from the globe segmented as described.) Fourth, the length of the MR muscle was measured from the origin near the orbital apex to the insertion through the point of tangency on the globe. Given these measurements, we computed the expected sideslip through steps of trigonometric calculations described by Robinson. ${ }^{5}$ We followed his suggestion that the bend at the insertion, because of the width of the muscle, be corrected by multiplying the cosine of vertical rotation angle to the vertical shift of the insertion. After such correction, the vertical translation of the MR insertion was then proportioned for a point on the muscle according to the proximity of the point to the insertion. In particular, we computed the expected amount of vertical shift at the part of the muscle on the plane tangential to the posterior pole of the globe and compared it with what was observed in the real MRI data for each individual subject.

\section{Results}

\section{Sideslip of the MR Muscle during Changes in Vertical Eye Position}

The MR muscle was identified in MRI images in all subjects. An example is shown in Figure $2 a$, in which the images are shown as a composite of two MRI data sets, one acquired while the eye was in abduction-supraduction and the other in abduction-infraduction. In Figure $2 \mathrm{~b}$, the composite was made between adduction-supraduction and adduction-infraduction. The two volumes in each figure were registered to remove the effect of head motion between scans, as described, and superimposed on top of each other. Eye-in-supraduction data were red, and eye-in-infraduction data were green. Thus, structures that retained their positions between the two scans are shown in yellow (i.e., red plus green), whereas those that shifted are visible as either red or green, depending on the contrast of the material and the direction of the shift. For example, the lens appears green and red in the composite images for up and down gazes, respectively, because it is darker in the MRI images than the surrounding material, (e.g., aqua humor in the anterior chamber). The axial slice of the orbit (Figs. 2a, 2b, top 
FIGURE 2. (a) Two sets of MRI volumes are shown as a color composite; one acquired with the eye in abduction-supraduction (red) and the other in abduction-infraduction (green). The positions of five sagittal slices shown on the right-hand side are marked by two white vertical lines in the axial (top left panel) and coronal (two bottom left panels) images. Only the most temporal (image 1) and nasal (image 5) sagittal slices are indicated for clarity. The positions of the coronal slices are at the white horizontal lines in the axial image-short ones for the image in the middle and long ones for the image at the bottom. White vertical lines in the sagittal images mark the location of the coronal slice in the right middle panel. The MR muscle and a part of the superior oblique muscles are denoted; the latter is seen in red because of taut contraction during infraduction. The position changes of the eyelid and the underlying soft tissues are reflected by green and red near the palpebral fissure (asterisk). (b) Similarly, the MR muscle is shown in composite images of two MRI volumes, one with the eye in adduction-supraduction and the other in adduction-infraduction. Markings on the figure are the same as in (a).
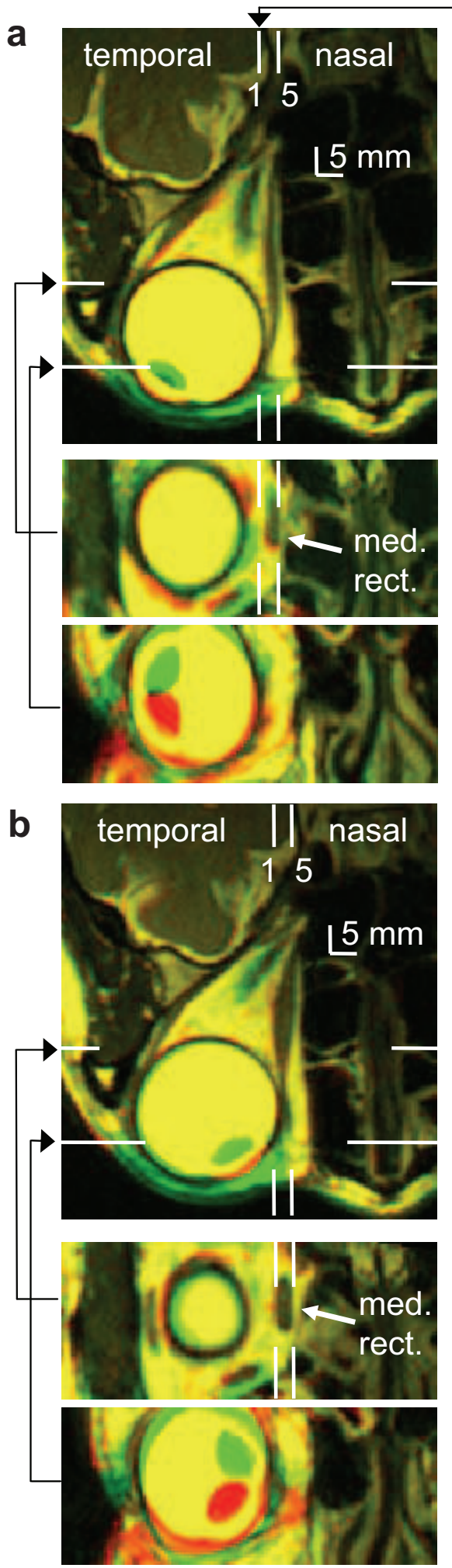
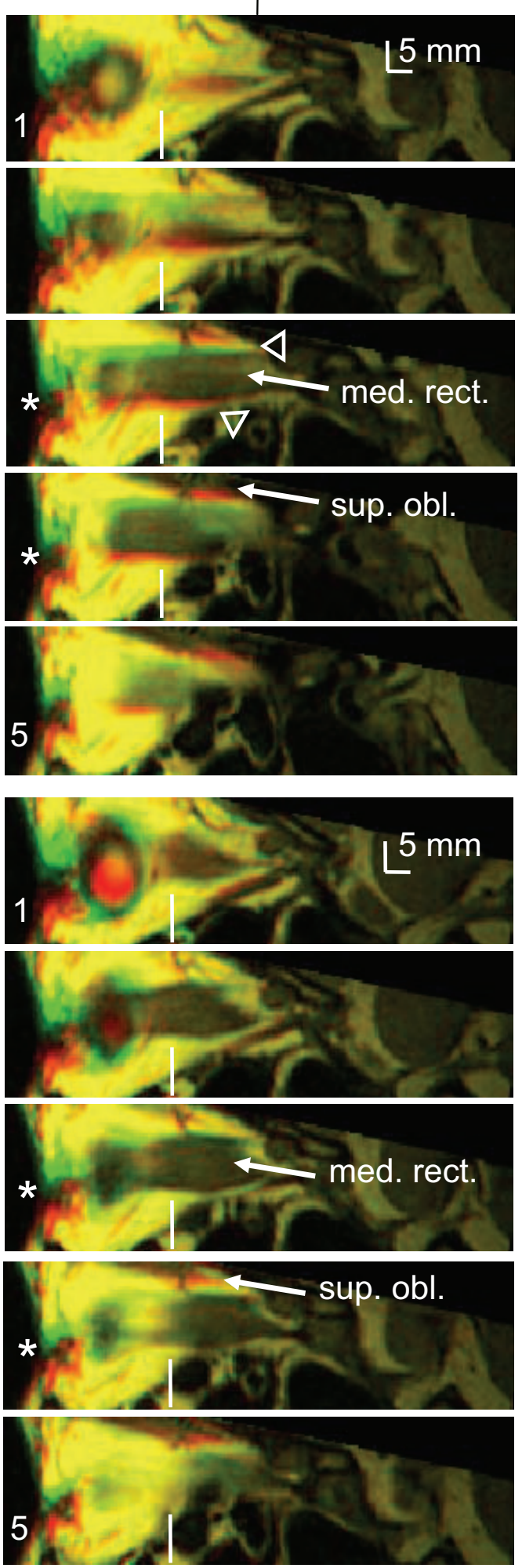

left) cut through the MR lengthwise, whereas the coronal slices taken through the lens show the muscle at cross-sections. Five sagittal slices in the right panels show the MR longitudinally at $0.84-\mathrm{mm}$ steps from the global side of the muscle (image 1) to the orbital side (image 5). A series of coronal slices shown in Figure 3 are cross-sections of the muscle at $1.68-\mathrm{mm}$ steps, starting from just in front of the equator of the globe to close to the apex.
When the eye moved between two tertiary positions in abduction (Figures $2 \mathrm{a}$ and $3 \mathrm{a}$ ), green and red shadows were observed at the top and bottom portions of the MR, indicating a vertical shift of the muscle belly. The shadows were clearly visible in sagittal, longitudinal sections of the muscle (Fig. 2a, open triangles) and in cross-sections (Fig. 3a). The shadows were smoothly graduated through the entire course of muscle path, with no abrupt change in width (Fig. 2a, sagittal slices), 
a
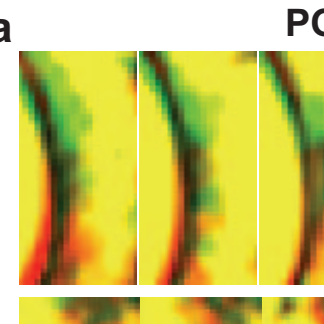

POT
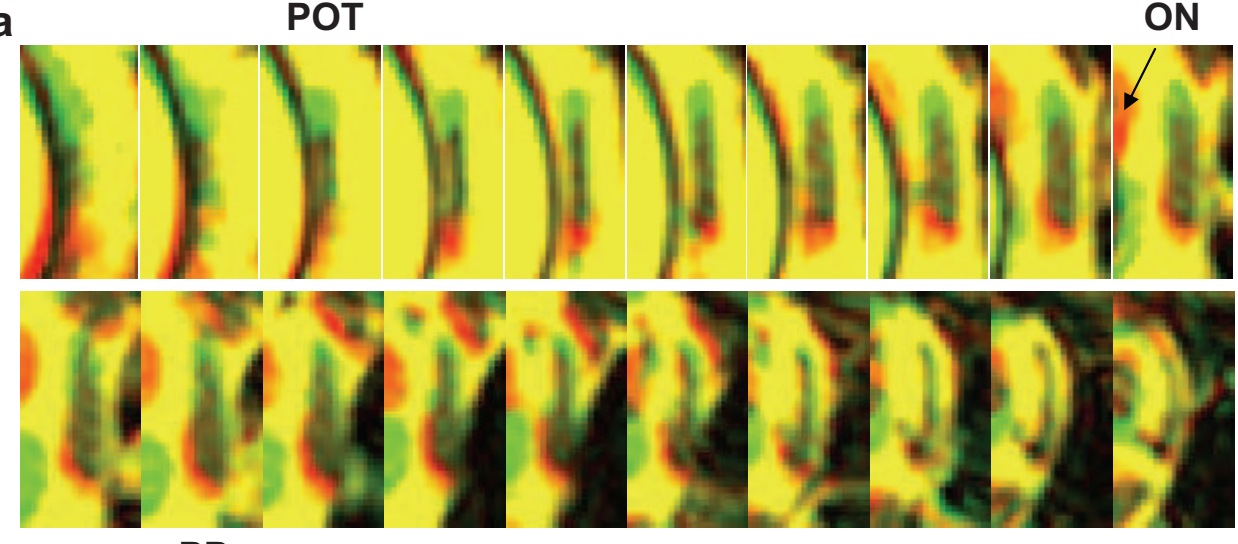

PP

b

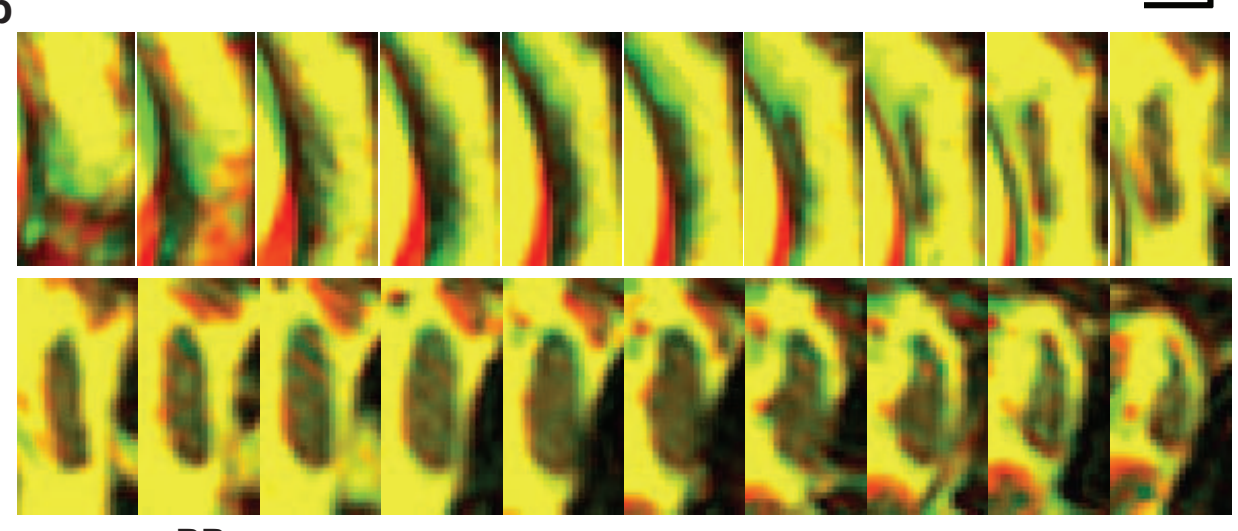

PP

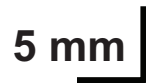

and no inflection point was found between the posterior pole of the globe and the point of tangency (Fig. 3a, POT).

In contrast, when tertiary positions in adduction were compared (Figs. 2b, 3b), the same muscle was shown in yellow, indicating that the muscle maintained its position despite the change in vertical eye position.

Additional examples of the vertical sideslip are shown in Figure 4 from two subjects who exhibited the largest (Fig. 4a) and smallest (Fig. 4b) sideslips.

\section{Vertical Sideslip of the MR Depending on Horizontal Eye Position}

Vertical sideslips of the muscle near the posterior pole of the globe were $1.5 \mathrm{~mm}$ on average when the eye was in $31.2^{\circ}$ abduction for $48.8^{\circ}$ vertical rotation (Fig. 5, circles on right). In contrast, vertical sideslip was only $0.2 \mathrm{~mm}$ for a comparable vertical rotation of $47.1^{\circ}$ when the eye was adducted by $39.0^{\circ}$ (Fig. 5, circles on left). The correlation between the amount of observed sideslip and horizontal eye position was statistically significant with the slope greater than zero (slope $0.0182 \mathrm{~mm} / \mathrm{deg} ; 95 \%$ confidence interval (CI) at $P<0.05,0.0097-0.027$; for the regression, $R^{2}=$ $0.7504, F=25.0543, P=0.0012$ ).

\section{Observed Sideslips and Predictions According to the Restricted Shortest-Path Model}

Sideslips predicted by the restricted shortest-path model were calculated from measurements of the muscle length, globe radius, and eye positions in individual MRI data (see Materials and Methods). Results are plotted in Figure 6 (open circles), along with actual observations (filled circles, as in Fig. 5).
Predicted amounts of vertical sideslips from the shortest-path model were, on average, $2.4 \mathrm{~mm}$ for the abduction data and $0.75 \mathrm{~mm}$ for adduction, larger than the observed value of 1.5 $\mathrm{mm}$ and $0.2 \mathrm{~mm}$, respectively.

These differences may be attributed to a restraining force exerted by the orbital fat and connective tissue networks surrounding the entire length of MR. ${ }^{9,10}$ In addition, the difference might have arisen from an elastic connective tissue structure that attaches the MR to the globe like a portal or a scaffold at and around the insertion, as suggested in the restraint shortest-path model by Miller and Robinson. ${ }^{6}$ The histology/anatomy near MR insertion is complex. ${ }^{11,12}$ The Tenon capsule is thick, anterior to the global equator (thickest over the MR insertion), and continuous with the intermuscular membranes. ${ }^{12}$ Some muscle fibers of the orbital layer insert into the capsule, which invests the muscle as the muscle sheath or "the pulley sleeve" (McNeer KW, et al. IOVS 2005; 46:ARVO E-Abstract 5721). ${ }^{13}$ Thus, the elastic capsule over the insertion may exert a stabilizing force near the insertion against the slippage between the muscle and the sclera during globe rotation and, in effect, may move the functional insertion to a more posterior location.

To compare relative contributions by the distributed restraint and the elastic capsule in reducing the vertical sideslip, we modeled the first factor (distributed restraint by surrounding tissue) as a multiplicative reduction of predicted sideslips and the second (the elastic coupling between the muscle and the globe) as a posterior relocation of the functional insertion. The best fit of this model to the observed data (shown as $x$ 's in Fig. 6) was obtained with a multiplicative reduction by 0.66 and an effective insertion at $1.3 \mathrm{~mm}$ posterior to that in the 
a

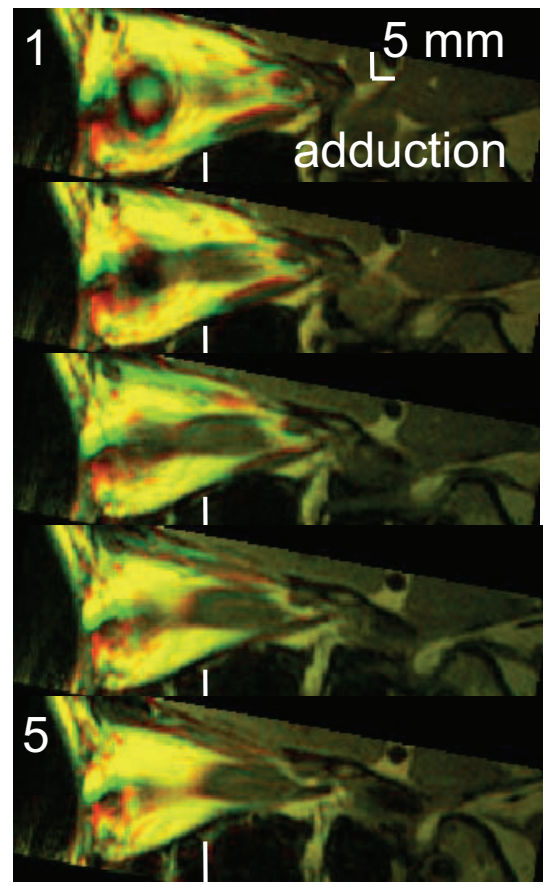

b

FIGURE 4. Sagittal slices through the medial rectus are shown for two additional subjects who showed the largest (a) and the smallest (b) sideslip. Shown are composite images of two MRI volumes with the eye in adduction-supraduction (red) and adduction-infraduction (green) in the left columns and with the eye in abduction-supraduction (red) and abduction-infraduction (green) in the right columns. Slices were obtained as in Figure 2, with the most lateral and most medial slices indicated by numbers 1 and 5, respectively. White vertical lines in the slices mark the location of a perpendicular plane that meets the posterior pole of the globe tangentially. White arrows indicate the observed vertical sideslips of the MR muscle.

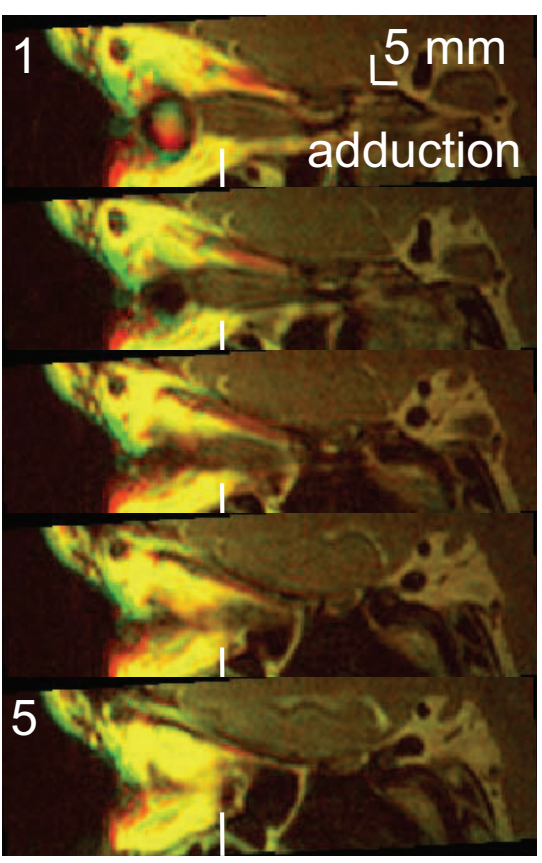

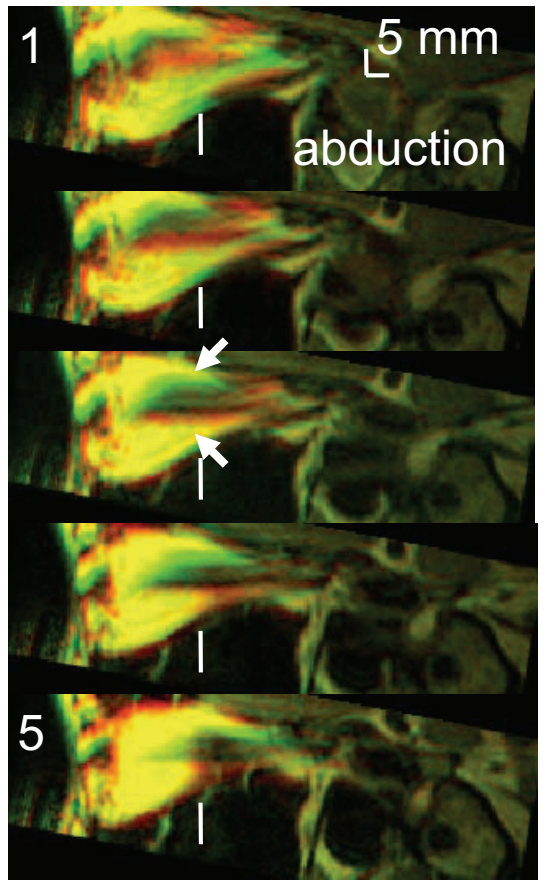

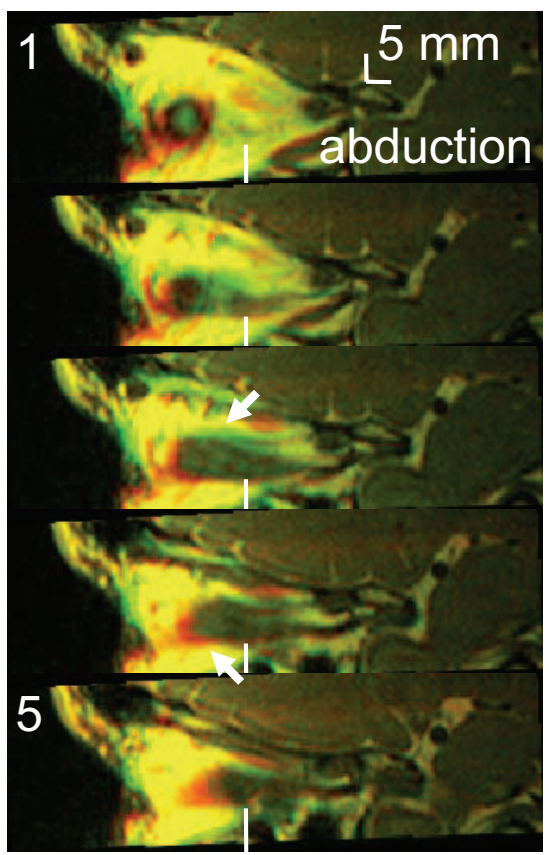

original calculation. These values seem plausible given mechanical conditions in the orbit in vivo.

\section{Discussion}

Contrary to previous reports, ${ }^{2,3}$ we observed that the MR indeed shifted sideways during vertical eye movements because of the high spatial resolution achieved in our study. Furthermore, we found that the amount of shift varied as a function of horizontal eye position, which was in accordance with the restrained shortest-path model, taking Listing's law of ocular kinematics into account.

Given the current findings, an alternative interpretation, in support of the pulley hypothesis, is that pulleys may reduce rather than abolish sideslip of the MR. Such an ad hoc inter- pretation poses difficulties in two aspects. First, the reduction of sideslip was not enacted by a localized restraint. No inflection by such a restraint-i.e., a pulley-was observed in the images. Instead the reduction appeared to be distributed along the whole course of the muscle path. Second, the fact that the sideslip was larger when the eyes were in abduction was contrary to the idea that a pulley held the muscle, even if it did so imperfectly. The MR is in least tension during abduction. Therefore, if it were restrained by a pulley at all, the sideslip would have been smallest here, and an inflection would have been observed. In fact, the sideslip was larger in abduction than in adduction.

Vertical sideslips demonstrated in our study must have occurred under distributed resistance from the surrounding tissue, such as the orbital fat and septal meshwork of connective 


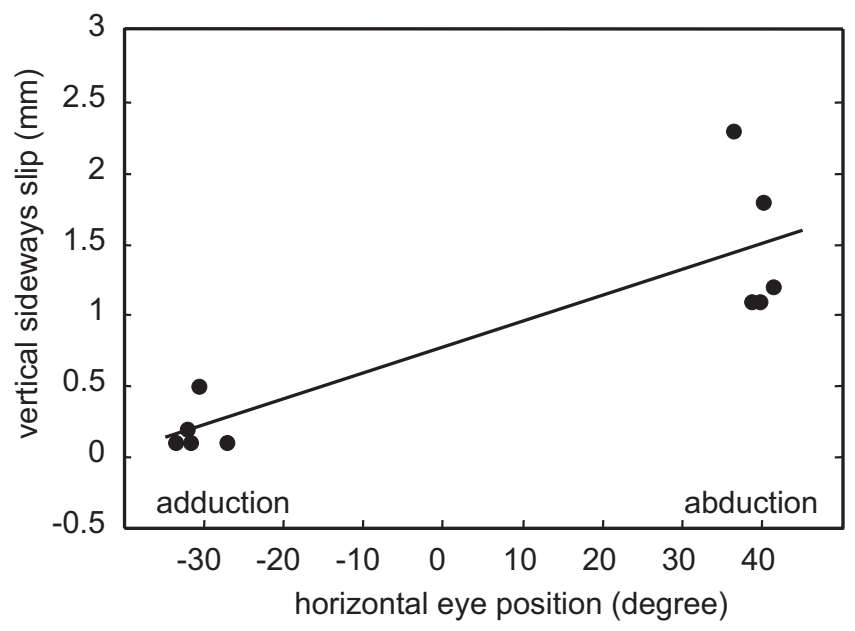

FIGURE 5. Sideslips observed in individual subjects are plotted as a function of horizontal eye position. For comparison with predictions according to the shortest-path model, see Figure 6.

tissue, ${ }^{9,10}$ rather than under localized restraint as the pulley model suggested. The amount of shift was graduated over the muscle length with no discernible inflection point, as was well demonstrated when the eye was in abduction and the shift was maximal. Since the MR is fully relaxed in abduction, a pulley would have restricted the muscle from sideslipping by making it bend around the pulley, but we did not observe this.

The revision of the shortest-path model by Miller and Robinson $^{6}$ considered elastic connective tissue coverings, at and around the insertional end of the muscle, that reduce the slippage of muscle against the sclera (hence, the restraint shortest-path model). This is clearly different from the pulley concept in that the coverings move with the globe, whereas the pulley is supposed to maintain its position in the orbit despite the rotations. A more recent active pulley hypothesis claims that the pulley may move in the direction parallel to the muscle length, but it still precludes any shifts perpendicular to it. ${ }^{1}$ The distinction between these two kinds of connective tissue restraints may perhaps be clearer when one thinks of the former (i.e., covering restraints over the insertion) as changing the functional insertion, whereas the latter (i.e., pulleys) assumes a change in the functional origin.

\section{Other Evidence against the Pulley Hypothesis}

Detailed examinations of the tissue surrounding the MR insertion have recently produced evidence incompatible with notions proposed in the pulley hypothesis. The orbital layer of the MR, as well as the global layer, inserts directly into the sclera. ${ }^{11}$ Moreover, the insertion is anterior to that of the global layer, ${ }^{11}$ in stark contradiction to the idea that the orbital layer myofibers insert into "the pulley sleeve" located posterior to the global layer insertion and "actively adjusts the pulley location." The "pulley sleeve," consisting of Tenon's capsule ensheathing the muscle and serving as the muscle sheath on the global surface, is too fragile on the global surface of the muscle to withstand the mechanical stress exerted in stabilizing the muscle. ${ }^{12}$ Furthermore, the pulley bands that were thought to stabilize the pulley sleeve with respect to the orbital wall were like a leash rather than a spring. ${ }^{14}$ They exerted negligible force against perturbation until distortions were extreme and thus could not possibly maintain the precise position of the pulley sleeve with respect to the orbit. Consistent with the findings of all these studies that questioned the pulley hypothesis, the current data provide in vivo evidence against the hypothesis by demonstrating that the medial rectus muscle is free to sideslip when required to take the shortest path from the anatomic origin to the insertion.

\section{Evidence Supporting the Pulley Hypothesis?}

The pulley hypothesis gained some popularity from mathematical reasoning that the pulleys might simplify the computation of $3 \mathrm{D}$ ocular kinematics by making the plant commutative for $3 \mathrm{D}$ rotations from the perspective of a controller, such as the neural circuitry in the brain, which controls the kinematic and dynamic aspects of eye movements. ${ }^{15}$ Although never a proof for the presence of the pulleys, this idea raised the popularity of the pulley hypothesis because the mechanical arrangements of the ocular plant alone might then be sufficient to implement Listing's law without help from neural processing. However, the enthusiasm for pulleys should be tempered by the realization that not all types of eye movement obey the law. If pulleys cannot always relieve the brain from the burden of complex computations, there is no real merit in invoking them. The computation may get more complicated because of the pulleys for types of movement that do not obey the law. In fact, the pulley idea runs counter to evolutionary wisdom in that it might decrease the work by the brain for evolutionarily new types of eye movements, such as saccades and smooth pursuit, at the cost of more work by the brain for evolutionarily older and presumably more basic forms of eye movements, such as angular vestibulo-ocular reflexes, which do not obey the law. ${ }^{7}$ Adding a complicated anatomic structure to make more primitive and earlier tasks more difficult does not make much sense.

More recently, observations from a few neurophysiological investigations were interpreted as supportive for the pulleys, which we think is at best tangential to the controversy over the pulley hypothesis. The observations were that activities of motor neurons in the abducens nuclei were not modified by vertical eye positions to reflect Listing' law. ${ }^{16}$ Yet microstimulation of the abducens nerve evoked eye movements in accor-

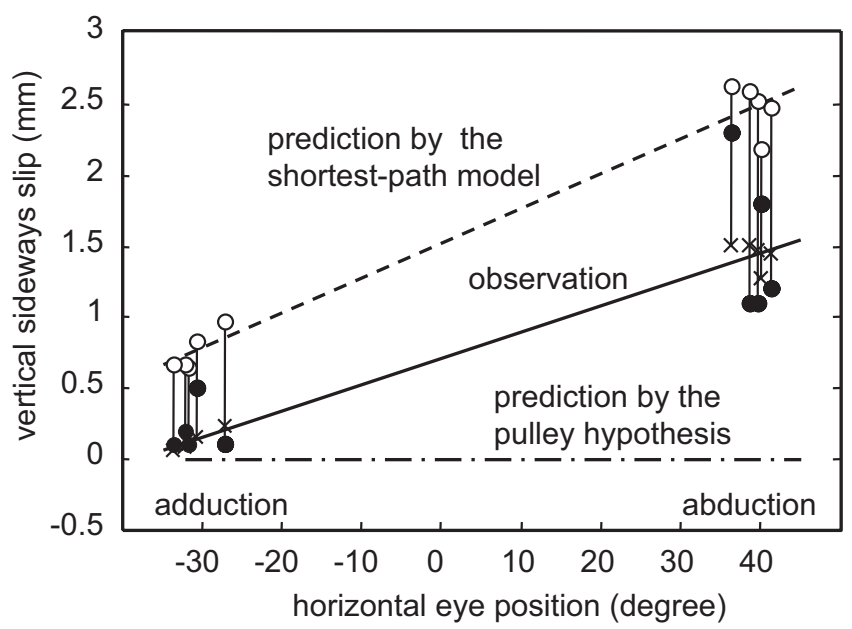

Figure 6. Sideslips observed in individual subjects are plotted as a function of horizontal eye position (solid circles), along with predictions according to the restricted shortest-path model (open circles) and values $(x$ 's) that best fit the restrained shortest-path model to the observed values with a multiplicative reduction and posterior relocation of the effective insertion. Observed and predicted amounts of sideslip for the same subjects are linked by a vertical line. For a pair of eye positions with similar horizontal direction, the difference in vertical deviations and the mean of horizontal deviations were used to calculate the prediction by the traditional model. Solid line: regression among the observed values. The slope of the regression $(0.018 \mathrm{~mm} /$ deg; $P<0.05$; CI, 0.0097-0.027) was significantly different from the prediction according to the pulley hypothesis (i.e., zero), but not from that by the shortest-path model (slope $0.025 \mathrm{~mm} / \mathrm{deg}$; dotted line). 
dance with Listing's law. ${ }^{17}$ These results were taken to mean that eye movements consistent with Listing's law occur by mechanisms distal to the motor neurons, in particular the connective tissue pulleys. However, if one wants to find out how Listing's law or, equivalently, the half-angle rule is implemented in horizontal eye movements, as the authors of the two studies did, the abducens neurons and the lateral rectus muscle are the last place to look because implementing the law for horizontal eye movements requires changing the rotation axis vertically; for this, the lateral rectus muscle is an insignificant player. The lateral rectus does indeed rotate the globe in the horizontal direction, but it is other muscles, particularly the cycloverticals, that have to adjust their tensions so that the rotation axis, lying vertically for the horizontal eye movements, is tilted by an appropriate amount, back or forth, depending on whether the eye is elevated or depressed, respectively. Thus, to find neural signature of Listing's law for horizontal eye movements, one should study cyclovertical muscles and neurons controlling them, not the lateral rectus or the abducens nucleus.

All oculorotatory muscles are active together in almost all eye positions. ${ }^{4}$ In fact, in a simulation study, ocular rotations in accordance with Listing's law were demonstrated when the ensemble of relative innervations was represented by a simple matrix formulation. ${ }^{18}$ Of particular interest for our arguments was the finding that stimulation-evoked eye movements also followed the law faithfully with no additional complicated assumptions, such as a bending of the muscle paths by pulleys (see the section on the effect of noncorresponding superposition in Ref. 18). These findings are in good agreement with the idea that Listing's law may be a consequence of the minimum effort principle in pseudo-inverse kinematics for oculomotor control, ${ }^{19}$ which in turn may arise from more fundamental properties of the neural system, such as the size principle, that stronger units are recruited at more extreme eye positions. ${ }^{20}$ Thus, Listing's law is likely a phenomenon that emerges from the coordinated performance of a population of neural controllers rather than a manifestation of mechanical properties of the oculomotor plant. The current observations concur with this view that complex $3 \mathrm{D}$ ocular kinematics must be implemented by neural signal.

Strabismus surgeons often either displace or abolish the possibility of any functional ability of a pulley, if it were as described by proponents of the pulley hypothesis. The current observations, along with other evidence against pulleys, ${ }^{11,12,14,21}$ are consistent with this clinical practice in that the straight path of the muscle obviates the necessity of worrying about inflections of the muscle by a pulley during surgery.

\section{References}

1. Demer JL. Current concepts of mechanical and neural factors in ocular motility. Curr Opin Neurol. 2006;19:4-13.
2. Simonsz HJ, Harting F, de Waal BJ, Verbeeten BW. Sideways displacement and curved path of recti eye muscles. Arch Ophthalmol. 1985;103:124-128.

3. Miller JM. Functional anatomy of normal human rectus muscles. Vision Res. 1989;29:223-240.

4. Boeder P. Co-operative action of extra-ocular muscles. $\mathrm{Br} \mathrm{J} \mathrm{Oph-}$ thalmol. 1962;46:397-403.

5. Robinson DA. A quantitative analysis of extraocular muscle cooperation and squint. Invest Opbthalmol. 1975;14:801-825.

6. Miller JM, Robinson DA. A model of the mechanics of binocular alignment. Comput Biomed Res. 1984;17:436-470.

7. Angelaki DE, Hess BJ. Control of eye orientation: where does the brain's role end and the muscle's begin? Eur J Neurosci. 2004;19: 1-10.

8. Collignon A, Maes F, Delaere D, Vandermeulen D, Suetens P, Marchal G. Automated multi-modality image registration based on information theory. In: Bizais Y, Barillot C, Di Paola R, eds. Proceedings of Information Processing in Medical Imaging. Dordrecht, The Netherlands: Kluwer Academic Publishers; 1995:263-274.

9. Koornneef L. Orbital connective tissue. In: Duane T, Jaeger E, eds. Biomedical Foundations of Opbthalmology. Vol 1. Philadelphia: Harper \& Row; 1983.

10. Schutte S, van den Bedem SP, van Keulen F, van der Helm FC, Simonsz HJ. A finite-element analysis model of orbital biomechanics. Vision Res. 2006;46:1724-1731.

11. Jaggi GP, Laeng HR, Muntener M, Killer HE. The anatomy of the muscle insertion (scleromuscular junction) of the lateral and medial rectus muscle in humans. Invest Ophthalmol Vis Sci. 2005; 46:2258-2263.

12. Ruskell GL, Kjellevold HI, Bruenech JR, van der WF. Double insertions of extraocular rectus muscles in humans and the pulley theory. J Anat. 2005;206:295-306.

13. Demer JL, Oh SY, Poukens V. Evidence for active control of rectus extraocular muscle pulleys. Invest Ophthalmol Vis Sci. 2000;41: $1280-1290$

14. van den Bedem SP, Schutte S, van der Helm FC, Simonsz HJ. Mechanical properties and functional importance of pulley bands or "faisseaux tendineux." Vision Res. 2005;45:2710-2714.

15. Quaia C, Optican LM. Commutative saccadic generator is sufficient to control a 3-D ocular plant with pulleys. J Neurophysiol. 1998; 79:3197-3215.

16. Ghasia FF, Angelaki DE. Do motoneurons encode the noncommutativity of ocular rotations? Neuron. 2005;47:281-293.

17. Klier EM, Meng H, Angelaki DE. Three-dimensional kinematics at the level of the oculomotor plant. J Neurosci. 2006;26:2732-2737.

18. Haustein W. Considerations on Listing's law and the primary position by means of a matrix description of eye position control. Biol Cybernet. 1989;60:411-420.

19. Warren PA, Porrill J, Dean P. Consistency of Listing's law and reciprocal innervation with pseudo-inverse control of eye position in 3-D. Biol Cybernet. 2004;91:1-9.

20. Dean P, Porrill J, Warren PA. Optimality of position commands to horizontal eye muscles: a test of the minimum-norm rule. $J$ Neuropbysiol. 1999;81:735-757.

21. Dimitrova DM, Shall MS, Goldberg SJ. Stimulation-evoked eye movements with and without the lateral rectus muscle pulley. J Neuropbysiol. 2003;90:3809-3815. 